\title{
Challenging Methods and Results Obtained from User-Generated Content in Barcelona's Public Open Spaces
}

\author{
Montserrat Pallares-Barbera ${ }^{1(\bowtie)}(\mathbb{D})$, Elena Masala $^{2}$ (D), \\ Jugoslav Jokovic ${ }^{3}$ (D), Aleksandra Djukic ${ }^{4}$ (D), and Xavier Albacete ${ }^{5}$ (D) \\ ${ }^{1}$ Universitat Autònoma de Barcelona, Bellaterra, Spain \\ montserrat.pallares@uab.cat \\ ${ }^{2}$ SiTI - Higher Institute on Territorial Systems for Innovation, Turin, Italy \\ elena.masala@polito.it \\ ${ }^{3}$ University of Nis, Niš, Serbia \\ Jugoslav.Jokovic@elfak.ni.ac.rs \\ ${ }^{4}$ University of Belgrade, Belgrade, Serbia \\ adjukic@afrodita.rcub.bg.ac.rs \\ ${ }^{5}$ University of Eastern Finland, Kuopio, Finland \\ xavialmar@gmail.com
}

\begin{abstract}
User-generated content (UGC) provides useful resources for academics, technicians and policymakers to obtain and analyse results in order to improve lives of individuals in urban settings. User-generated content comes from people who voluntarily contribute data, information, or media that then appears in a way which can be viewed by others; usually on the Web. However, to date little is known about how complex methodologies for getting results are subject to methodology-formation errors, personal data protection, and reliability of outcomes. Different researches have been approaching to inquire big data methods for a better understanding of social groups for planners and economic needs. In this chapter, through UGC from Tweets of users located in Barcelona, we present different research experiments. Data collection is based on the use of REST API; while analysis and representation of UGC follow different ways of processing and providing a plurality of information. The first objective is to study the results at a different geographical scale, Barcelona's Metropolitan Area and at two Public Open Spaces (POS) in Barcelona, Enric Granados Street and the area around the Fòrum de les Cultures; during similar days in two periods of time - in January of 2015 and 2017. The second objective is intended to better understand how different types of POS' Twitter-users draw urban patterns. The Origin-Destination patterns generated illustrate new social behaviours, addressed to multifunctional uses. This chapter aims to be influential in the use of UGC analysis for planning purposes and to increase quality of life.
\end{abstract}

Keywords: User-generated content $\cdot$ Big data $\cdot$ Twitter $\cdot$ Public open spaces • Spatial analysis 


\section{Introduction}

The implications of User Generated Content are changing the daily life of people. Everyone is involved, but some institutions have stored the data and the power to exploit them. We can contribute to make this power more democratic and available to improve the life of people. This chapter has the goal to do it. The objective of this chapter is to deepen the study on user-generated data (UGD, User Generated Content, UGC) for exploring new methodologies that could support and improve the understanding of spatial patterns for urban planning and design of open spaces in urban areas. This chapter aims at taking a step forward form current literature. It provides, on the one hand, three methods for social science analysis using Big Data; and, on the other hand, it expects to motivate the possible outcomes of UGD coming from three Information and Communication Technologies (ICT) platforms into policy driving strategies, where results can be used to improve quality of life. This chapter examines the spatial pattern of UGD in three public open spaces in Barcelona: Enric Granados Street (Carrer Enric Granados), Forum (Fòrum de les Cultures) and Barcelona Metropolitan Area; and the spatial patterns generated by weekday and by hourly interval in order to study the further insides of how users construct different configuration in visiting a city. Methodologically, we have used two different platforms (Twitter and WAY app). Twitter, the web social network is currently used by 284 million of monthly active users (Twitter Inc. 2015). Twitter provides large amounts of data georeferenced. Although only a part of the entire urban population uses Twitter, the remaining data are a useful source to analyse the location of people in the city are and why there is more activity-clustering in places vs. others. The second platform is the app WAY Cyberparks, an application developed by DeustoTech Mobility within the COST Action TU1306 - CyberParks (Bahillo et al. 2016, 2015; Masegosa et al. 2015). This GPS-based platform focuses on the use of ICT for understanding and collecting data on the use of urban open spaces.

The possibility to communicate worldwide through common smartphones opened up new perspectives and interaction pathways to individuals, who very quickly responded with a massive use of social network platforms and applications. Since most smartphones include Global Positioning System (GPS), if activated, outgoing data from each device can be geo-referenced. Thus, the large amount of data can be geo-located with a time reference. The consequence is the constant production of "Big Data" (Targio et al. 2015; Snijders et al. 2012) which can be overlaid on maps and analysed by mathematical, semantic techniques (Language detection API 2016), and by means of spatial analysis methods. Substantial works on the nature of geography have discussed the spatial pattern formed by many purposes of analysis, since the classical works of Hartshorne (1939), Harvey (1963), Friedman and Alonso (1964), Berry (1973), and Chorley and Haggett (1965) among many others till nowadays. Their relevant research on the organization and settlement of people and activities in the human world, the regularity of distribution brought significant and worthy results which made huge advancements in Geographical Thought and society. In 2018, UGC 
brought a new paradigm in the study and analysis of spatial patterns in Human Geography. Related problems are more associated to find appropriate models and methodologies to draw a good statistical sample from the population, to depurate the data, to discriminate the data size and to analyse consistently the results.

Case studies are very specific and different POS in Barcelona. Enric Granados is located at the urban centre, in the "Cerdà's Eixample" (Cerdà enlargement) of Barcelona (Pallares-Barbera, Badia and Duch 2011). It is a residential lively, dense street with services and amenities; Forum is located at the end of the Diagonal, very close to the 22@Barcelona neighbourhood (Casellas and Pallares-Barbera 2009). It is mainly a complex of buildings and cement plazas built for the 2004 Forum of Cultures exhibition. For the most part, locals use Enric Granados Street, while Forum's facilities are used for large meetings, conventions and festivals; otherwise it is an unoccupied space.

This chapter is formulated in different sections. Section 2 analyses the state of the art regarding uses and applications of UGC by different institutions, firms and policy makers. Section 3 describes the case studies. Section 4 focusses on data and methodology, while Sect. 5 is about results and discussion of them. Conclusions, provided in Sect. 6, aim to offer insides of expected future developments for the integration of UGC with new methodologies within contemporary spatial planning processes.

\section{Background and Related Work}

The availability of big and open data is nowadays offering new opportunities for rethinking the human behaviour and its impact on Earth. Since these data is often georeferenced, its overlaying on maps provides useful information on particular organization and structure of spatial systems. Therefore, urban planning, and design, transport planning, social sciences and human geography can benefit from their use.

Although a large number of applications and experiments constellates the landscape of research, the academic research has barely yet found a specific own pathway within these huge opportunities. However, relevant academic work is coming out as well as public and private bodies have begun to use big data for their own development; for example, NOAA Big Data Project, or DataKind for the treatment of trees in NYC. A concise overview on the state of the art on the use of geolocated user-generated contents in spatial planning is meant to show analysis, applications and uses.

Cities have the pressure of millions of inhabitants to increase their quality of life. Through the introduction of ICT, cities try to cope population needs with strategies based on an integrative framework, including management, technology, governance and policy; a reunion of factors which some call smart city (Chourabi et al. 2012). The concept of smart city has not a clear consensus and nor a consistent understanding among academia and practitioners, but in this chapter, it will be used to refer to a city with massive implications of ICT. City information is collected by sensors, tools and applications and used on the city policy. Data-information relies on the diffuse use of 
devices for quantifying movements, environment quality and healthiness, and energy consumption. Private enterprises, such as the biggest technological companies like IBM, Cisco or Siemens are also interested on data collection and analysis for their own objectives and strategies.

Local institutions such as governments or urban planning firms use big data to organize exhibitions with different goals. Some of them are under the idea of crowdsourcing, in which citizens' digital footprint such as tweets or use of online municipal services help city planners improve city's design and functionality. For instance, the Chicago Architecture Foundation (CAF) partnering with IBM (Chicago Architecture Foundation 2014; IBM 2014; Snodgrass 2014) launched "Chicago: City of Big Data." Its goal was to open up a dialogue on the connection between big data and human lives and to show how spatial systems are impacted by this information, and how relevant each individual was to the design of their environment. The exhibition's dashboards were built with IBM's City Forward platform, a free web-based "civic resource that enables people to visualize and interact with city data" (Kokalitcheva 2014). The methodological basis for selecting data sources used two central criteria: the data's availability at a large enough scale; and its capability to communicate valuable insights about the city's needs and flows. Then, they used complex geographic information systems (GIS) and web-based tools to compile and visualize the data in effective ways. Data sources consisted of over 18 million Twitter data points, with only timestamp and location attributes, which turned into dynamic and "interactive models of city's human activity." (Kokalitcheva 2014). On the broad objective of "explor(ing) the emergence of the database as a framework for cultural and political thinking and the effects of datafication of the world.", the Big Bang Data exhibition (Spain) (organised by the Centre de Cultura Contemporània de Barcelona (CCCB) and Fundación Telefónica 2015) took under consideration different topics about city' shape and layout on the basis of different kind of data.

Academic literature based on Twitter-use patterns has developed at different geographical scales. The spatial pattern of social networks and the threshold of their activities have been analysed at urban scale across three cities in order to produce an inter-urban analysis (Bawa-Cavia 2010). Diverse methods, such as attempts to aggregate the activity on the network onto a grid $(400 \times 400 \mathrm{~m})$ of dots representing the 'walkable' cells showed the threshold walking distances in urban areas (O'Sullivan and Morral 1996). At wider scale, human flows are gathered, extracted and analysed in geolocated social media data through different instruments. An example is FlowSampler, an interactive interface for visual analysis of flows in geolocated social media data. It adopts a graph-based approach to infer movement pathways from spatial point type data and expresses the resulting information through multiple linked visualizations to support data exploration (Chua et al. 2014). Additionally, it allows characterizing places in base the "density" of arc traces and keywords within the local groups. Big data also helps to study prescription planning such as to plan routes of buses and subways to satisfy public demand in several cities around the world (Fisher 2012). 
Through the spatial analysis of UGD, we come to understand that some cities are far more active early in the morning, while others show higher activity at night or on weekends (Neuhaus 2011). The Centre for Advanced Spatial Analysis (CASA) at University College London (UCL) Twitter-use monitored several cities over a week to determine patterns in temporal activities and learn about spatial networks. Other analyses on UGD and available data from social networks - Twitter, Facebook, Flickr and Instagram - captured information about citizens' feelings, citizens' uses, citizens' comfortability and conditions of problematic situations. Funded by the German Research Foundation, the "Urban Emotions" project (Heidelberg and Kaiserslautern Universities) tested UGD by checking whether the emotions measured correlate with the subjective assessments in the social media. "People as Sensors" used measures emotions and stress levels, such as "unsafe bike paths, traffic jam stress, frightening underpasses" (Heidelberg University 2014). Therefore, measurements of citizens' emotional responses to their environment were made with by the use of sensors, similar to a wristwatch "that allows us to measure skin conductance, body temperature and variations in heart rate that change, for instance, when someone is startled" (Resch et al. 2015). Differently, but also tracing emotions, the exploration of how people express excitement online show a regular pattern; in which as much higher levels of excitement and more intense the flurry of messages in the collective, the shorter the messages become (Senseable City Lab and Ericsson 2015; Szell et al. 2014). Emotional bursts become faster and more impulsive online than offline. Many associated questions and outcomes become still unanswered, such as: "Are people doing this independently, or in response to seeing other short messages? Are we following the herd? Could we use these insights to learn more about financial bubbles by measuring more impulsive, less rational responses? And can we design better communication services?" (Lanzerotti et al. 2013).

Nevertheless, cautions have to be taken in considering the use of big data. There are many positive aspects on big data analysis, but many fears can be raised too. The obvious one is the intrusion on in the private sphere of our lives. In addition, putting data culture at the centre of decision-making and on the way of interpreting the world opens up many possibilities and involves numerous risks. The main danger of datacentrism is that it encourages the idea that whatever the problem, the answer lays in data. This important discussion escapes the goal of this chapter.

\section{Case Studies}

The several big data methodologies presented in this chapter are better understood if different geographical scales are used for analysis. Besides the metropolitan area of Barcelona, two very diverse morphological and social POS are chosen: Enric Granados and Forum (Fig. 1). 


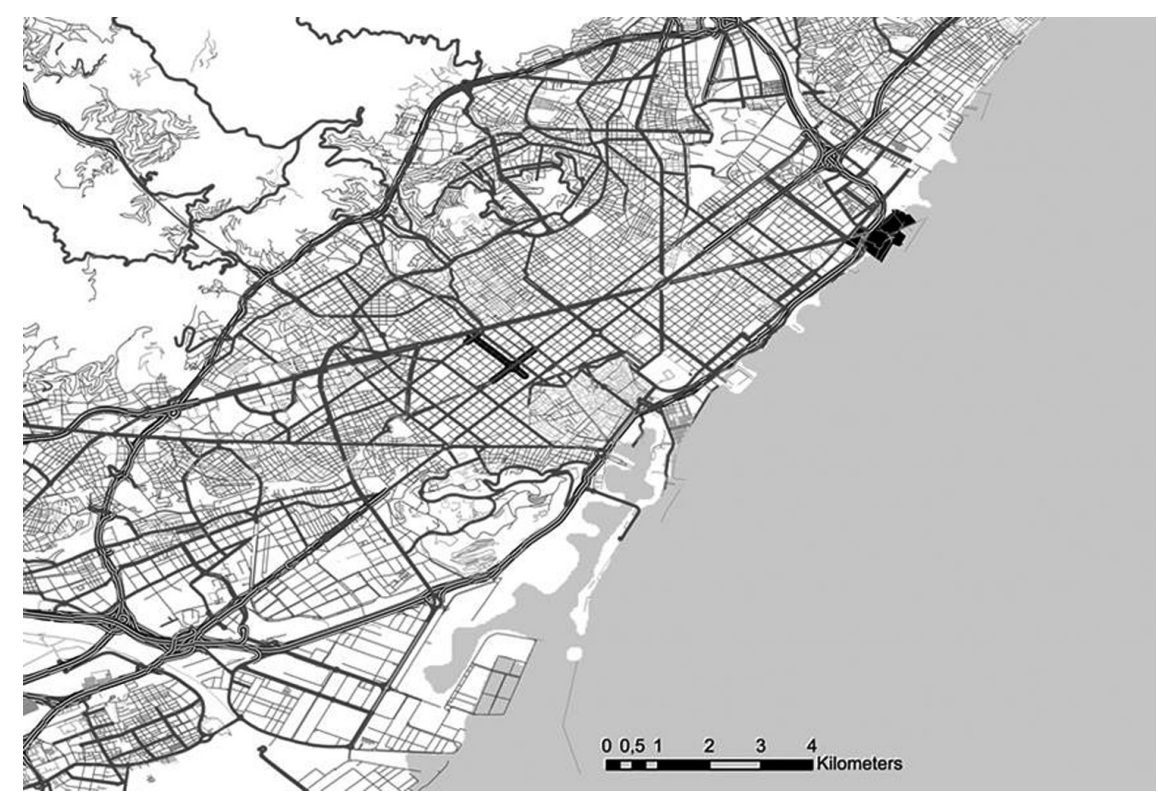

Fig. 1. Location of two case study areas in Barcelona

Enric Granados is within the consolidated historical city; and connects the Diagonal Avenue with a foundational building of the University of Barcelona. Differently from Rambla de Catalunya and Passeig de Gràcia, which are parallel to it, the Enric Granados width is slightly more than $20 \mathrm{~m}$ and hosts several leisure activities mostly for neighbours and local people. The urban transformations that followed the Olympic Games in 1992 changed the role of this street in the urban hierarchy. From a traditional car-oriented road, it became a pedestrian that is a user-oriented space. This was made possible by modifying the car mobility of the street.

The Forum rises on the old industrial Poblenou, an urban district that was renewed during the end of the twentieth, beginning of the twenty-one century; and hosted the Universal Forum of Cultures (2004) (Casellas, Dot-Jutgla and Pallares-Barbera 2012). Forum is where the Diagonal Avenue meets the Mediterranean Sea. The new character of the area is pointed by a number of new architectures, with building, skyscrapers designed by very famous architects. Nevertheless, the functional connectedness of the whole area is inexistent. The large paved esplanade provides a sense of incompleteness with no seats, nor trees, nor shadows.

\section{Data and Methodology}

The several methods used in this chapter are based on geo mapping. Focus is placed on intensity and concentration of users in open public spaces. Data was collected using 3 different ICT methodologies in 3 periods of time in Barcelona. First, Twitter data was collected by SiTI (Higher Institute on Territorial Systems for Innovation, Torino) during 
the period between January the 12th and 18th, 2015. Second, Twitter data was compiled using TSE (Tweeter Search Engine) application in January from the 13th to the 20th, 2017. Third, WAY app gathered data in November 27, 2014 (COST Action 1306 2014), with a selected group of users moving in and between the two case study areas.

\subsection{Twitter Data Information}

Twitter is used by only $23 \%$ of the total population in the U.S.A. Its data-base is open for free use and can provide very large amount of records and information, including attributes on time and location of each single tweet (geo-referenced points and the given radius are settled only when sender is geotagged (Poblete et al. 2011; Krumm, Davis and Narayanaswami 2008; Haining 2003)). Each message can be downloaded and stored on a personal database through Twitter REST API (Twitter REST API 2016). Some of the characteristics of the database include: the text, time and date, the nick-name of the sender, the eventual name of the receiver, and the latitude and longitude from sender (Table 1).

Table 1. Data records of a single tweet

\begin{tabular}{c|c|c|c|c|c|c}
\hline D & Message & User from & User to & Date & Lat & Lon \\
\hline $\begin{array}{c}\text { Calvin } \\
\text { http://t.co/14oG7mPxwE }\end{array}$ & $\begin{array}{c}\text { Outside - } \\
\text { Harris }\end{array}$ & albasancho95 & fjdh & $\begin{array}{c}\text { Wed Jan 07 } \\
08: 55: 20 \quad+0000 \\
2015\end{array}$ & 41.47267 & 2.27114 \\
\hline $\begin{array}{c}\text { Nos aventuramos a las } \\
\text { rebajas... Tengo miedo xD }\end{array}$ & $\begin{array}{c}\text { zaidas- } \\
\text { phyxiated }\end{array}$ & & $\begin{array}{c}\text { Wed Jan 087 } \\
08: 55: 18 \quad+0000\end{array}$ & 41.52691 & 2.229489 \\
\hline & \#cyberparks \#TU1306 & e_frola & & $\begin{array}{c}\text { Fri Jan 09 } \\
10: 29: 08 \quad+0000 \\
2015\end{array}$ & 41.50982 & 2.229217 \\
\hline
\end{tabular}

For each single user, it is possible to collect other specific data, which can be joined to previous one. This data is listed below.

- User ID

- Name

- Location

- Info given by the user

- Following

- Creation data

- Time zone

- Language

- Status message and date

- Follower ID. 


\subsection{Methodology for Data Mining for Twitter by SiTI, January 12-18, 2015}

The total amount of records was 72,257 Tweets, of which 67,251 (93.07\%) were georeferenced. Among these, only 2,802 (4.17\%) records were from users who passed across one or both of the two case study areas within the city. The following six steps were used: first, we elaborated Twitter data in Excel; second, we imported Twitter data in ESRI Arc Map; third, we used information about time and coordinates of data from Cyberparks App to trace plausible paths of single users (or group of users); fourth, we worked the information about time and coordinates of tweets to trace plausible paths of single users (or group of users) within specific areas; fifth, we used information about time and coordinates of tweets to trace plausible paths of single users (or group of users) who pass through specific areas; and sixth, we used tweets information about languages, presence of tweets for large periods (to differentiate local users from tourists or visitors), keywords, to identify different kinds of densities within specific areas.

\subsection{Methodology for Data Mining for Twitter Using Twitter Search Engine (TSE), January 13-20, 2017}

To collect, storage, process and analyse Twitter data in January 13-20, 2017 (1517000 tweets per day), we employed Twitter Search Engine (TSE) (Dinkic, Jokovic and Stoimenov, 2016). It is a micro-blogging platform that provides a rich collection of real-time commentaries which are on the Twitter REST API, which storage tweets sent the previous week within a given radius. TSE allows collection and storage of data for unlimited periods of time; it offers a display, analysis and execution of complex geospatial queries of the data stored in the database. These queries are executed with the help of relational geospatial functions offered by MySQL database. TSE functions are correlated in terms of interrelationship between two objects determined with georeferenced points. TSE has the option of drawing a polygon on the map of Google by using Google Maps JavaScript API. This polygon site must be within the area for which information was collected. TSE also has the ability to detect the language using web service Language Detection API (Language detection API, 2016). This API has the capacity to detect 160 different languages and to offer 5.000 requests for free on daily basis ${ }^{1}$. Each request must contain text and API key. The request example can be found at the link ${ }^{2}$. Language Detection API produces results in *.json format. Response contains array of language candidates. Each object contains language code, confidence score and is reliable - true/false (Table 2).

\footnotetext{
${ }^{1}$ To use this API, one has to register on their website using a valid email address. Then, the user receives an API key; which can be used for client application and depending on the needs sends GET or POST requests to server.

$2 \mathrm{http} / / /$ ws.detectlanguage.com/0.2/detect?q=buenos+dias+señor\&key=demo.
} 
Table 2. Language Detection API - Response example

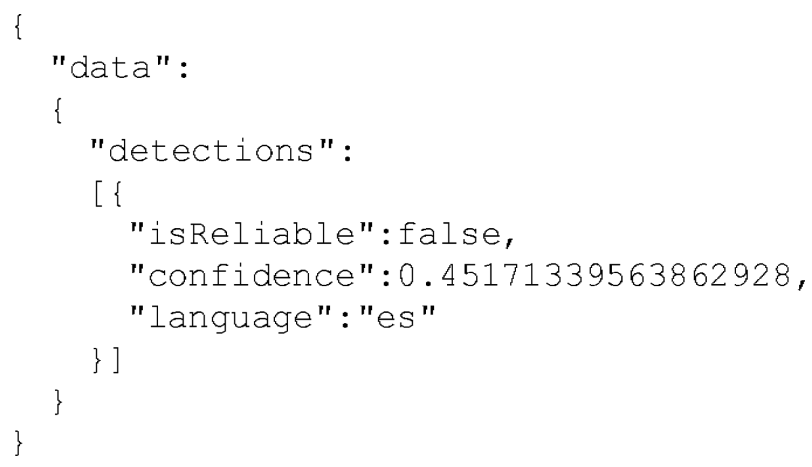

\subsection{Methodology for Data Mining for WAY App, November 27, 2014}

The WAY app (Deusto Tech Mobility 2014) is a GPS-based platform that, differently from Twitter data, registers the users' movements twice a second. The result is a point database which allows building up a timeline with the full path travelled by users, with continuity and precision. The app is conceived as a Volunteered Geographic Information (VGI) platform where users collaborate for producing information. In particular, it collects data for better understanding the use of public open spaces in order to improve their production and their relevance to sustainable urban development. It collects also data on the user such as age, sex, education, job, the distance from home and from working place, and the reason of being in a public space, such as walking, running, reading, kids or pets. The main limit of WAY data is given by the fact that the person who generates the data, the user, is conscious that he/she is producing data for some specific analyst task. Therefore, it is necessary to consider that this awareness can alter the veracity of the data. Geovisualization of these data is done by importing data from WAY and converting the *.json file into a readable file for ESRI ArcMap.

\section{Results and Discussion - Maps and Visualizations}

Several spatial patterns have arisen from the planned methods analysing the use of Twitter and WAY app. Although these initial analyses can be developed further, their opportunities for specific additional improvements are open in future research; where data comparisons, fitness of the methodology and so on are to be implemented to meet diverse research structural questions. 


\subsection{Tweet Patterns in Barcelona Metropolitan Area, January 12-18, 2015 and January 13-20, 2017}

The Origin/Destination (O/D) matrix and the points of twitter messages show the centrality of the city, respect to its hinterland (Fig. 2a, b). City diversity is perceived by the number of user languages (22 (2015) and 120 (2017)) (Table 3).

(a)

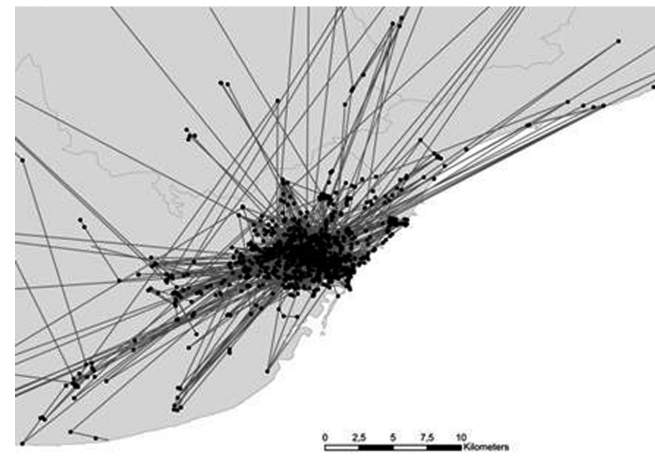

(b)

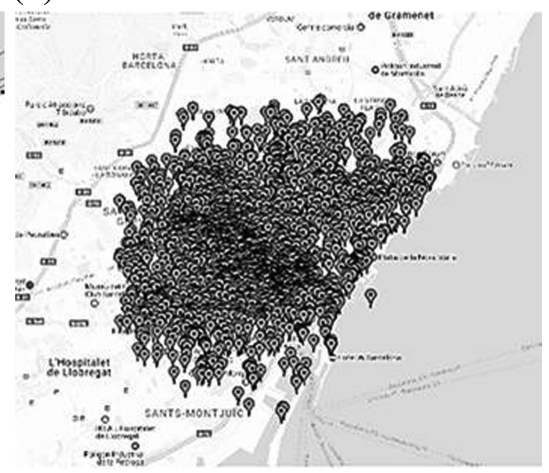

Fig. 2. Twitter messages. Barcelona Metropolitan Area (BMA) (a), and Barcelona City $(\mathrm{BCN})(\mathrm{b})$

Table 3. Data collected for Barcelona

\begin{tabular}{|c|c|c|}
\hline Type of analysis & January $12-18,2015$ & January 13-20, 2017 \\
\hline Number of tweets & 67251 & 117017 \\
\hline Number of users & 9823 & 10412 \\
\hline Number of retweets & - & 20208 \\
\hline Number of likes & - & 51989 \\
\hline Number of applications & - & 45 \\
\hline Number of languages & 22 & 120 \\
\hline
\end{tabular}

\subsection{Tweets by Weekdays in Barcelona Metropolitan Area, January 12-18, 2015, January 13-20, 2017}

There are variations in the use of the space by weekdays, and between years 2015 and 2017. 2017 has almost doubled the number of tweets of 2015; it might correspond to the increase of tweeter users globally (Table 4, Fig. 3). In 2015, the day with less number of tweets is Thursday, while Monday is the day with more activity. On 2017, on Friday 17 there are fewer tweets than the rest of days. It is obvious that for statistics analysis testing more data values would be required; these two weeks are not representative of others. 
Table 4. Tweets by day

\begin{tabular}{l|c|c|c|c}
\hline \multirow{2}{*}{ Day } & \multicolumn{2}{|l|}{ January 12-18, 2015 } & \multicolumn{2}{l}{ January 13-20, 2017 } \\
\cline { 2 - 5 } & Number of tweets & \% of tweets & Number of tweets & \% of tweets \\
\hline Monday & 10535 & 15.67 & 17295 & 15.00 \\
\hline Tuesday & 9500 & 14.13 & 17122 & 15.00 \\
\hline Wednesday & 9410 & 13.99 & 16542 & 14.00 \\
\hline Thursday & 8649 & 12.86 & 16927 & 14.50 \\
\hline Friday & 9464 & 14.07 & 15681 & 13.00 \\
\hline Saturday & 10474 & 15.57 & 16533 & 14.00 \\
\hline Sunday & 9219 & 13.71 & 16917 & 14.50 \\
\hline Total & 67251 & 100 & 117017 & 100 \\
\hline Mean & 9607 & & 16717 & \\
\hline
\end{tabular}

(a)

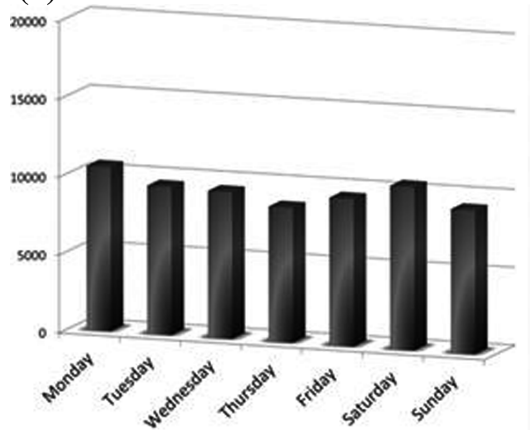

(b)

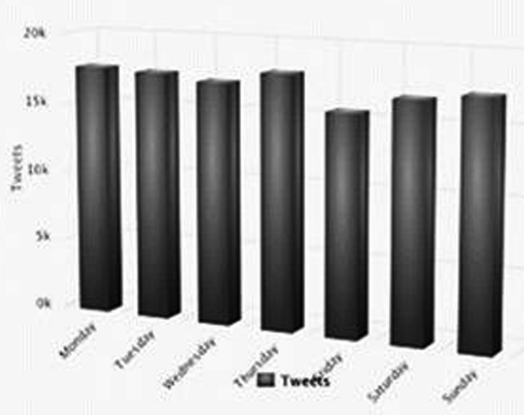

Fig. 3. Tweets by days in the period January 12-18, 2015 (a), and January 13-20, 2017 (b)

Further visualization for the period in 2015 shows subsequent tweets connected by semi-circular arches, whose radius is proportional to the spatial distance which separates each couple of tweets (3D arches) (Fig. 4). On Wednesday 14, 2015 arches are shorter than other days, then activities are concentrated in a smaller space within the city centre; or Sunday 18, the activity around Plaça de Catalunya is comparatively less, shops are closed.

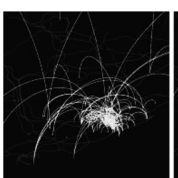

Mon 12

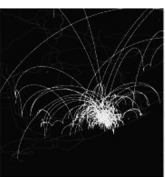

Tue 13

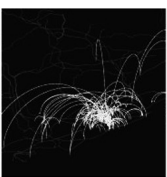

Wed 14

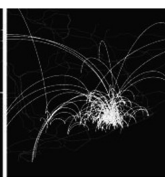

Thu 15

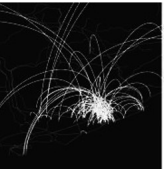

Fri 16

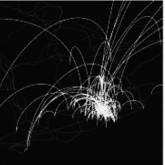

Sat 17

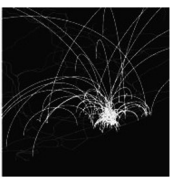

Sun 18

Fig. 4. Distribution of subsequent tweets by each same user, January 12-18, 2015, Barcelona 


\subsection{Tweets Per Hour, January 12-18, 2015, January 13-20, 2017}

City life changes during the day. Seen by number of tweets, the tendency is to increase during the evening, decreases at night, and start increasing in the morning (Table 5 and Fig. 5). Tweets are concentrated around axes that pass Rambla; and most of tweets (75\% in 2017) have been sent from 12.00 until 24.00, which relates to working time and street activity (Table 5).

Table 5. Percentage of tweets by time interval

\begin{tabular}{l|c|c}
\hline Interval & January 12-18, 2015 & January 13-20, 2017 \\
\hline $0-3$ & $6.54 \%$ & $4.28 \%$ \\
\hline $3-6$ & $2.52 \%$ & $1.71 \%$ \\
\hline $6-9$ & $8.85 \%$ & $7.95 \%$ \\
\hline $9-12$ & $12.77 \%$ & $13.85 \%$ \\
\hline $12-15$ & $15.42 \%$ & $17.64 \%$ \\
\hline $15-18$ & $15.05 \%$ & $18.05 \%$ \\
\hline $18-21$ & $19.68 \%$ & $20.38 \%$ \\
\hline $21-24$ & $19.17 \%$ & $16.14 \%$ \\
\hline
\end{tabular}

\subsection{Results Provided by WAY App During November 27, 2014}

The results of the WAY app provide more detailed perception by users at a micro-scale level. In one sense, WAY data is continuous and is following users' path; while tweets are diffuse in space and time dimensions (Figs. 6 and 7). In another sense, WAY data is concentrated only in the two case studies, whereas Twitter data is sparsely located around the city (Fig. 8). Finally, WAY offers very detailed information on places and on single user's movements, while Tweets provide general information about hierarchical functions in places.

(a)

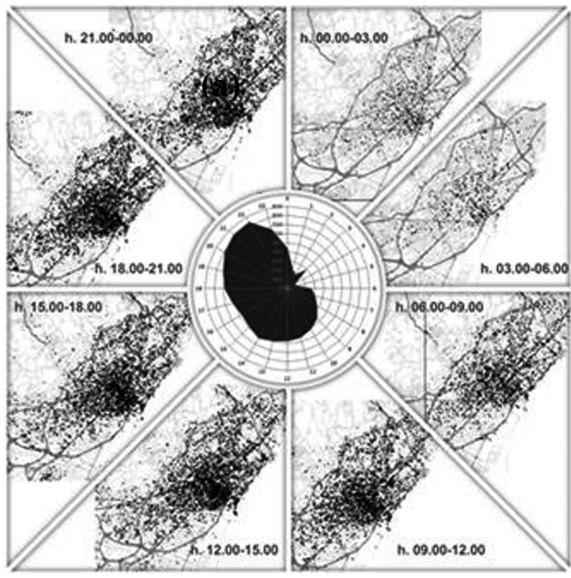

(b)

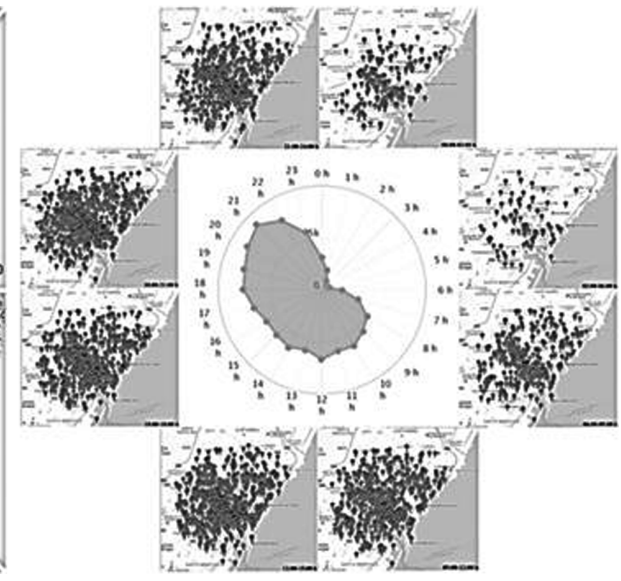

Fig. 5. Tweets by time interval, in the period January 12-18, 2015 (a) and January 13-20, 2017 (b) 


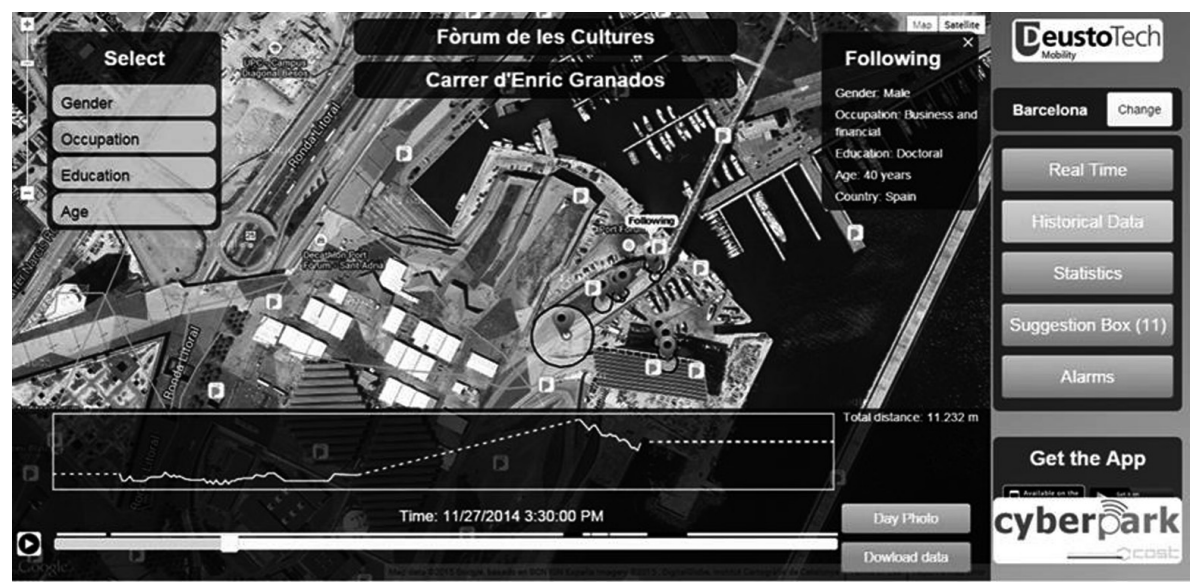

Fig. 6. Visualisation of data gathered in Forum through WAY app

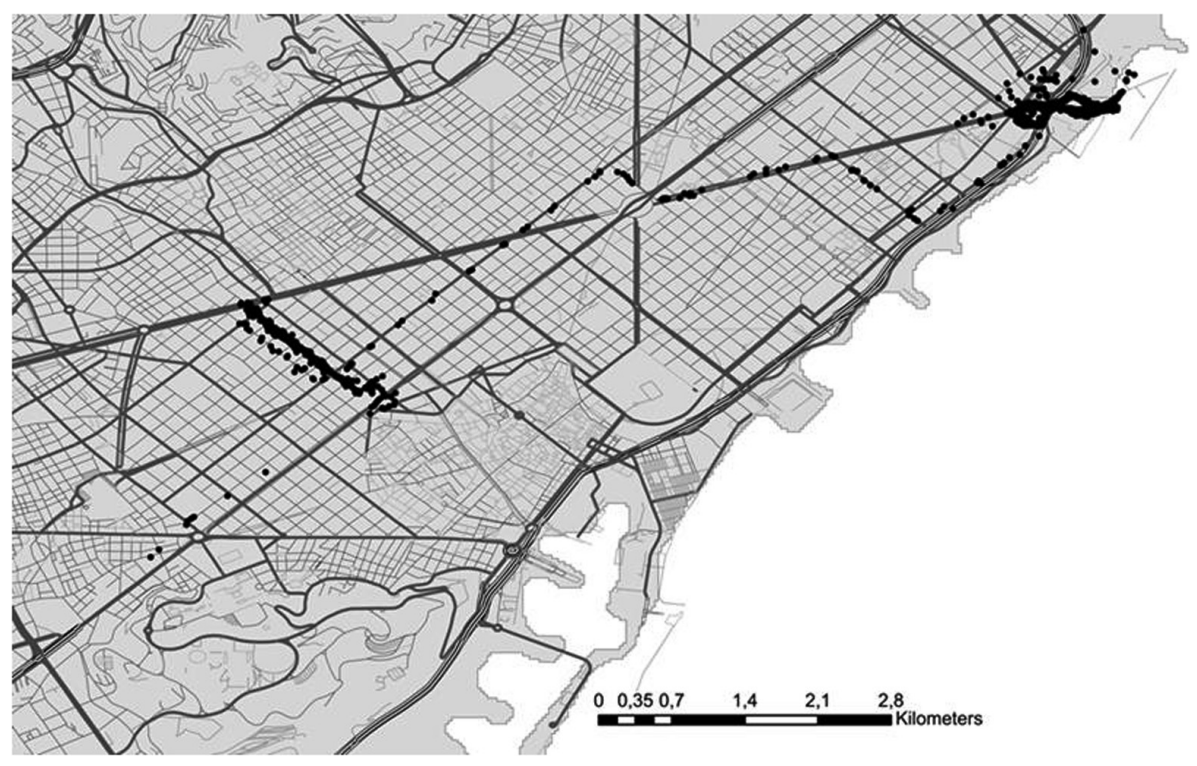

Fig. 7. Data collected by the WAY app in Enric Granados, Forum and in the way between both areas 


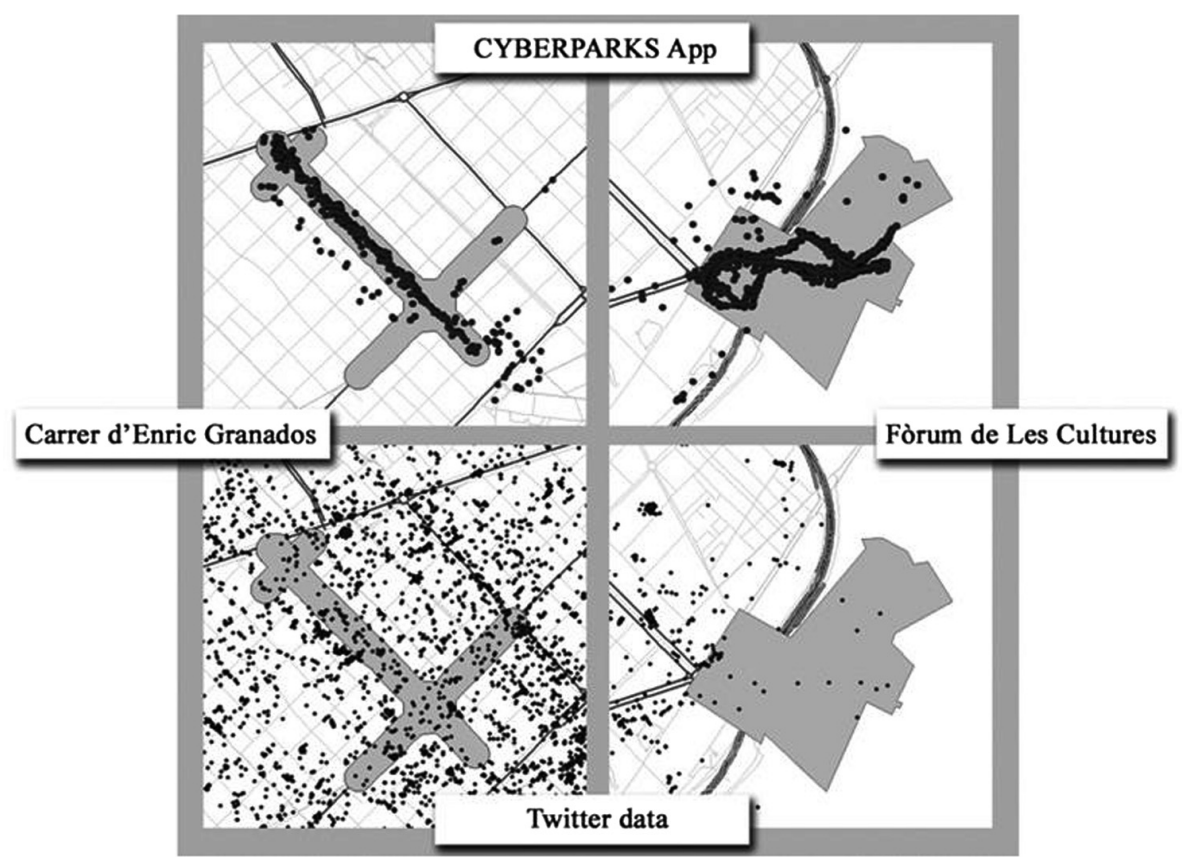

Fig. 8. WAY and Twitter data compared, November 24, 2014

\section{Conclusions and Future Developments}

The overview on the state of the art shows how spatial planning is facing a transition presenting new challenging scenarios. The trend led by the smart city opened the spatial planning to logic of numbers and data. City-sensors as well as UGC systems constantly provide huge amounts of data which can be used to supply the citizens' demands. In this context, two main branches are emerging. The first branch is a dataoriented and technology-driven approach, which makes use of quantities for assessing and justifying decisions. Eye-catching visualizations are the most evident outputs of such an approach, where analysts and statistics prevail on the human experience of professionals and experts.

The second branch uses new technologies to improve human abilities and it is mostly used to support the decision and policy-making processes. Particular efforts are spent in understanding how data can support can be complementary to the traditional approach, providing new insights on spatial issues. The debate on the use of big data is nowadays shifting from a technology-driven vision towards a more human dimension, introducing the concepts of people friendliness and a human-to-human approach (Melis et al. 2014). To achieve this social vision, the collaboration between different experts such as urban planners and data analysts and designers appears essential in order to guide them towards relevant questions and policy issues (Goodspeed 2012). Simple visualizations offer opportunity for both horizontal and vertical communication. 
From a technical point of view, some considerations are necessary for improving future map production. First of all, paths created using subsequent tweets are just possible routes and probably not the real ones covered by users. A method for dealing with this issue is necessary, and different research is being undertaking. Secondly, the use of Twitter data is most suitable for larger scale than the micro-urban areas. However, the continuity along space and over time provide important element for considering their hierarchical position.

In this chapter, the analysis was limited to visualisation and maps; it did not considered semantics, the written content of the tweets (however, a method for experiencing has been suggested); statistical testing has not been performed. Nevertheless, the humble objectives have been stated and proved. We know that integration of new methodologies within spatial planning processes are very relevant, such as in the data collecting and information; defining goals, objectives and strategies and implementation of users' participation through crowdsourcing.

\section{References}

Bahillo, A., Aguilera, T., Álvarez, F.J., Perallos, A.: WAY: seamless positioning using a smart device. Wirel. Pers. Commun. 94(4), 2949-2967 (2016)

Bahillo A, Diez, L.E., Perallos, A., Falcone, F.: Enabling seamless positioning for smartphones. In: Proceedings of Conference XXX Simposium Nacional de la Unión Científica Internacional de Radio (URSI), Pamplona, Spain (2015)

Berry, B.J.L.: The human consequences of urbanisation: divergent paths in the urban experience of the twentieth century, vol. 3, Macmillan, Basingstoke (1973)

Bawa-Cavia, A.: Sensing the urban: using location-based social network data in urban analysis. Presented at 1st Workshop on Pervasive Urban Applications PURBA 2011, San Francisco, USA (2010)

Casellas, A., Dot-Jutgla, E., Pallares-Barbera, M.: Artists, cultural gentrification and public policy. Urbani izziv 23(Suppl. 1), S104-S114 (2012)

Casellas, A., Pallares-Barbera, M.: Public sector intervention in embodying new economy in inner urban areas: the Barcelona experience. Urban Stud. 46(5-6), 1137-1155 (2009)

Centre de Cultura Contemporània de Barcelona, CCCB \& Fundación Telefónica (2015). http:// bigbangdata.cccb.org/en/sec-exhibition/. Accessed 12 Jan 2015

Chicago Architecture Foundation (CAF) (2014). http://bigdata.architecture.org/. Accessed 12 Jan 2015

Chorley, R.J., Haggett, P.: Trend-surface mapping in geographical research. Trans. Inst. Br. Geogr. 37(4), 47-67 (1965)

Chourabi, H., et al.: Understanding smart cities: an integrative framework. system science (HICSS). In: 45th Hawaii International Conference on Hawaii, USA, pp. 2289-2297. IEEE (2012)

Chua, A., Marcheggiani, E., Servillo, L., Vande Moere, A.: FlowSampler: visual analysis of urban flows in geolocated social media data. Presented at: International Conference on Social Informatics, Barcelona, Spain (2014)

COST Action 1306: First working meeting at the geography department (Universitat Autònoma de Barcelona), Cerdanyola, Spain (2014)

Deusto Tech Mobility: Public open spaces monitoring tool (2014). http://www.costcyberparks.eu/. Accessed 12 Jan 2015 
Dinkic, N., Jokovic, J., Stoimenov, L.: Software application: Twitter search engine, developed at the University of Nis, Faculty of Electronic Engineering, during the Ph.D. course "Advanced topics in data and knowledge engineering”, Nis, Serbia (2016)

Fisher, E.: Paths through cities (2012). www.flickr.com/photos/walkingsf/sets/721576290 14750905/detail/. Accessed 11 Feb 2015

Friedman, J., Alonso, W.: Regional Planning and Development. The MIT Press, Cambridge (1964)

Goodspeed, R.: The democratization of big data. 27 February (2012). http://www.planetizen. com/node/54832. Accessed 2 Jan 2015

Haining, R.: Spatial Data Analysis: Theory and Practice. Cambridge University Press, Cambridge (2003)

Hartshorne, R.: The nature of geography: a critical survey of current thought in the light of the past (conclusion). Ann. Assoc. Am. Geogr. 29(4):413-658 (1939). www.jstor.org/stable/ 2561166. Accessed 2 Mar 2016

Harvey, D.W.: Locational change in the kentish hop industry and the analysis of land use patterns. Trans. Papers (Inst. Br. Geogr.) 33, 123-144 (1963). https://doi.org/10.2307/621004

Heidelberg University: Urban design with emotions. Press Release No. 166/2014 (2014). www. uni-heidelberg.de/presse/news2014/pm20140911_urban-design-with-emotions.html. Accessed 9 Jan 2015

IBM: A new blueprint: how chicago is building a better city with big data (2014). http:// people4smartercities.com/series/new-blueprint-how-chicago-building-better-city-big-data. Accessed 12 Jan 2015

Kokalitcheva, K.: How a swarm of data is helping Chicago re-map urban life (2014). http:// venturebeat.com/2014/05/14/how-a-swarm-of-data-is-helping-chicago-re-map-urban-life. Accessed 12 Jan 2015

Krumm, J., Davies, N., Narayanaswami, C.: User-generated content. IEEE Pervasive Comput. 7(4), 10-11 (2008)

Language detection API (2016). https://detectlanguage.com/. Accessed 15 Apr 2016

Lanzerotti, L., Bradach, J., Sud, S., Barmeier, H.: Geek cities: how smarter use of data and evidence can improve lives (2013). www.bridgespan.org/Publications-and-Tools/Performance-Measurement/Geek-Cities-Data-Improves-Lives.aspx\#.VLZ9BSvF9Ks. Accessed 13 Jan 2015

Masegosa, A., Bahillo, A., Onieva, E., López, P., Perallos, A.: A new optimization approach for indoor location based on differential evolution. In: Proceedings of Conference International Fuzzy Systems Association (IFSA), Gijón, Spain (2015)

Melis, G., Masala, E., Tabasso, M.: From the smart city to the people-friendly city: usability of tools and data in urban planning. In: Vesco, A., Ferrero, F., (eds) Social, Economic, And Environmental Sustainability In The Development Of Smart Cities, IGI global, pp. 363-381 (2014)

Neuhaus, F.: Twitter data - seeking spatial pattern (2011). http://urbantick.blogspot.it/2011/03/ twitter-data-seeking-spatial-pattern.html. Accessed 9 Jan 2015

Open street map community (2004). www.openstreetmap.org. Accessed 29 Dec 2014

O'Sullivan, S., Morral, J.: Walking distances to and from light-rail transit stations. Trans. Res. Rec. 1538, 19-26 (1996)

Pallares-Barbera, M., Badia, A., Duch, J.: Cerdà and Barcelona: the need for a new city and service provision. Urbani izziv 22(2), 122-136 (2011)

Poblete, B., Garcia, R., Mendoza, M., Jaimes, A.: Do all birds tweet the same? characterizing Twitter around the world. Presented at CIKM 2011 20th ACM Conference on Information and Knowledge Management (2011). www.ruthygarcia.com/papers/cikm2011.pdf 
Resch, B., Summa, A., Sagl, G., Zeile, P., Exner, J.-P.: Urban emotions-geo-semantic emotion extraction from technical sensors, human sensors and crowdsourced data. In: Gartner, G., Huang, H. (eds.) Progress in Location-Based Services 2014. LNGC, pp. 199-212. Springer, Cham (2015). https://doi.org/10.1007/978-3-319-11879-6_14

Senseable City Lab \& Ericsson (2015). http://senseable.mit.edu/tweetbursts/. Accessed 12 Jan 2015

Snijders, C., Matzat, U., Reips, U.D.: Big data: big gaps of knowledge in the field of internet science. Int. J. Internet Sci. 7(1), 1-5 (2012)

Snodgrass, N.: Chicago: city of big data. exhibition explores the digital age of urban design (2014). www.architecture.org/document.doc?id=1074. Accessed 12 Jan 2015

Szell, M., Grauwin, S., Ratti, C.: Contraction of online response to major events. PLoS one, 2(26) (2014)

Takhteyev, Y., Gruzd, A., Wellman, B.: Geography of Twitter networks. Soc. Netw. 34(1), 1-25 (2011)

Targio, I.A.H., Yaqoob, I., Anuar, N.B., Mokhtar, S., Gani, A., Khan, S.U.: The rise of big data on cloud computing: review and open research issues. Inf. Syst. 47, 98-115 (2015)

Twitter Inc. About Twitter, Inc. (2015). https://about.twitter.com/company. Accessed 4 Feb 2015

Twitter REST API (2016). https://dev.Twitter.com/rest/public. Accessed 15 Jan 2016

Open Access This chapter is licensed under the terms of the Creative Commons Attribution 4.0 International License (http://creativecommons.org/licenses/by/4.0/), which permits use, sharing, adaptation, distribution and reproduction in any medium or format, as long as you give appropriate credit to the original author(s) and the source, provide a link to the Creative Commons license and indicate if changes were made.

The images or other third party material in this chapter are included in the chapter's Creative Commons license, unless indicated otherwise in a credit line to the material. If material is not included in the chapter's Creative Commons license and your intended use is not permitted by statutory regulation or exceeds the permitted use, you will need to obtain permission directly from the copyright holder.

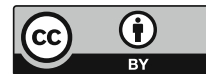

